\title{
Impact of Early-Life Antibiotic Use on Gut Microbiota of Infants
}

\author{
Wen Hong $Y^{\# *}$, Su Si T*, Yuan Zhen Y, Wang Xiu Y and Wang Guo Z \\ School of Life Science, Jiangsu Normal University, Xuzhou 221116, China \\ "Authors contributed equally
}

\begin{abstract}
The human gut has a vast number of bacteria, which play a critical role in human health. At present, it is accepted that antibiotics have been one of the most common drugs in the world and early-life antibiotic use is associated with increased risk for several diseases. Antibiotic use during infancy will induce imbalances in gut microbiota, which is called dysbiosis. Therefore, more and more researches have been known about the impact of antibiotics on gut microbiota of infants. Here, we discuss some effects of antibiotics on gut microbiota and health of infants, including four types: structure of gut flora, metabolic capacity, diversity and stability of gut microbiota, risk of diseases. We also profile antibiotic resistance gene carried by gut microbiota and mechanism of intestinal drug -resistant bacteria. This article will also help provide recommendations for antibiotic use during infancy.
\end{abstract}

Keywords: Gut microbiota; Infants; Antibiotic resistance; Antibiotic resistance gene

\section{Introduction}

Our microbiome, termed the second genome, comprises a vastly more significant number of cells and genes than those derived from the human gametes [1]. In healthy people gastrointestinal ecosystem, there are enormous and complicated bacteria, more than 10 trillion, called gut microbiota. Gut microbiota is often considered to be an important organ acquired by the human body, which is known as body's second brain [2]. It has been identified that our gut has nearly 9.88 million microbial genes [3]. Throughout the human lifetime, gut microbiota has a profound effect on all aspects of human health, such as protecting the host against pathogenic bacteria, promoting digestion and absorption of the host, drug metabolic and carcinogen, affecting the absorption and distribution of fat, regulating energy metabolism, regulating innate and adaptive immune systems and so on [4-10]. In a recent article, Vikram et al. reported that gut microbiota also promotes atherosclerosis [11]. And not only that, it has become evident that changes in the composition of the gut microbiota also have great physiological influence on people [12]. The type and number of gut microbiota are vulnerable to change by a variety of factors such as age, diet, antibacterial drugs and psychological pressure, causing imbalances in gut microbiota and diseases [refs]. Development of the intestinal microbiota in infants is characterized by rapid and large changes in microbial abundance, diversity, and composition [13,14]. Therefore, more and more attention has been given to the impacts of antibiotics on gut microbiota of infants. The gut of infants is sterile before they are born. At the time of birth, a variety of bacteria start breeding in infant gut. Originally, gut microbiota were mainly of Coliform, Enterococci and Clostridium. Five days later, Bifidobacteria occupied dominant positions. In neonates, the early development of the microbiota differs between antibiotic-treated and non-treated infants, but nothing is currently known about the longterm associations between lifetime antibiotic use and gut microbiota in infants. Furthermore, antibiotic resistance is of major public health concern globally and is expected to become an increasingly serious obstacle in the treatment of infections [15].

In this review, to better understand the impacts of early-life antibiotic use on gut microbiota and health of infants, we combine recent abroad pertinent literature. We describe the impacts of antibiotics from four aspects: (i) structure of gut flora, (ii) metabolic capacity, (iii) diversity and stability of gut microbiota, (iv) risk of diseases. We also discuss the potential effects of antibiotic resistance gene(s) carried by gut microbiota and investigate mechanism of intestinal drug resistant bacteria. This article aims to better understand effects of early-life antibiotic use on infants' gut microbiota and health take mechanism of intestinal drug-resistant bacteria into account and provide a reference for clinical treatment. Finally, the potential fields of further researches are prospected.

\section{Antibiotic use disrupts structure of gut flora and metabolic capacity}

In clinical treatment, antibiotics are a class of commonly used drugs. In recent years, experimental studies showed that antibiotics can destroy the structure of normal gut microbiota and change the functions $[16,17]$. In the study of Klemm and Dougan, they have found that antibiotics use led to remarkable changes in the structure of gut microbiota by phylogenetics analysis [18]. Antibiotics use during infancy is closely associated with the changes in the composition of gut microbiota which last for more than 6 months. To resolve the origin of gut microbiota and thoroughly research the possible impact of antibiotics use and other environmental factors on gut microbiota, a team of researchers from the Massachusetts General Hospital (MGH) and the Broad Institute selected a cohort of 43 U.S. infants and analyzed microbial development during the first 2 years of life systematically [19]. They identified multiple disturbances associated with antibiotic exposures, cesarean section, and formula feeding. What's more, experimental studies have proved that antibiotic exposures delayed microbiota maturation. Delayed microbiota maturation, as defined in healthy children, mirrors physiological disturbances in the host and occurs in mice exposed to antibiotics [20,21].

In a study at the University of Helsinki in Finland, Korpela et al.

${ }^{*}$ Corresponding author: Wen Hong Y, School of Life Science, Jiangsu Normal University, Xuzhou 221116, PR China, E-mail: wenhy@jsnu.edu.cn

Received September 25, 2017; Accepted October 12, 2017; Published October 19, 2017

Citation: Hong YW, Si TS, Zhen YY, Xiu YW, Guo ZW (2017) Impact of Early-Life Antibiotic Use on Gut Microbiota of Infants. J Microb Biochem Technol 9:227-231. doi: 10.4172/1948-5948.1000369

Copyright: @ 2017 Hong YW, et al. This is an open-access article distributed under the terms of the Creative Commons Attribution License, which permits unrestricted use, distribution, and reproduction in any medium, provided the original author and source are credited. 
investigated 142 Finnish children aged 2-7 years (median age 5 years) who attended the same day-care centers at the time of the study to understand how the use of specific antibiotics can affect the intestinal microbiota in different ways [15]. The $16 \mathrm{~S}$ rRNA sequencing data of the bacterial community obtained from the fecal samples showed that children's gut microbial community composition can clearly reflect the use of antibiotics and antibiotic use will slow down the development of microbial community $[22,23]$. Children who received macrolides, such as azithromycin and clarithromycin in early life had obvious changes in the gut microbiota composition and metabolic capacity. Specifically, the abundance of Actinobacteria, which included Bifidobacterium as the dominant genus, was reduced, whereas the abundances of the Gram-negative phyla Bacteroidetes and Proteobacteria were increased [24]. However, children who used penicillin didn't have such marked changes. Furthermore, the researchers found a positive correlation between overall lifetime antibiotic use and body mass index (BMI). The study results confirmed and extended previous results from mouse experiments and indicated that macrolide use may have detrimental effects on the developing microbiota of children, which may threaten the development of a healthy immune system and metabolism [20,2527]. Generally speaking, the intestinal microflora can recover within 1 year after a macrolide course. However, if the child is repeatedly used in early life, the microbial community will not fully recover.

\section{Antibiotic exposures impair the diversity and stability of gut microbiota}

The balance between gut microbiota and the host immune system ensures the host health and homeostasis [28]. Recent research of Bokulich et al. has demonstrated that antibiotic exposures, cesarean section and formula feeding will significantly diminish the diversity and stability of gut microbiota [19]. In collaboration with a team of Finnish researchers they have worked with for several years, the Massachusetts General Hospital team enrolled a group of 39 children in 3 years [29]. During the study period, they collected their fecal samples and found decreased microbial diversity and increased shortterm composition changes in the gut microbiota of antibiotic-treated children [30]. In this case, low-Bacteroides children were even more obvious. Whole-genome shotgun (WGS) sequencing analysis also showed that, in antibiotic-treated children, bacterial species were fewer and tended to be controlled by a single strain, which was very different from that of the children who had not been exposed to antibiotics. With the elapse of time, many samples analysis revealed that the gut microbiota of antibiotic-treated children could become less stable, particularly around the period of antibiotic therapy. It has been a fact that antibiotics intensified the dysbiosis [31].

Another, published in 2016 by Finnish researchers, reported that antibiotic use, and macrolide use in particular, was associated with a long-term reduction in microbial richness [15]. Antibiotic-treated children still did not reach the level of the control samples even 1224 months after the course. Some aspects of the microbiota, such as the abundance of Bifidobacterium and Bacteroides and macrolide resistance, normalized within 12 months after a macrolide course. However, the abundances of Collinsella and Lactobacillus remained reduced for up to 2 years after a macrolide course.

Infants, particularly those born prematurely, represent an interesting population because they received early and often extensive antibiotic therapy in the first months after birth [32]. Gibson et al. recently demonstrated that children who had been exposed to antibiotic therapy had a dramatical reduction in the diversity of their microbial population. Meropenem, ticarcillin-clavulanate and cefotaxime treatments were associated with decreased species richness; gentamicin and vancomycin had variable effects on species richness [33]. One of the key motivations of microbiome research is that the microbial population of early childhood is crucial to human health because the decreased diversity of gut microbiota will cause some allergies and autoimmune diseases [29].

\section{Antibiotic use increases the risk of diseases}

Some previous studies have shown that antibiotic exposure in children had been associated with increased risk of immune system diseases, such as inflammatory bowel disease, obesity, diabetes, asthma and allergies [34-37]. It is generally accepted that gut microbiota have close relationship to these disease [38]. Alterations in gut microbiota composition and bacterial metabolites have been increasingly recognized to affect host metabolism in metabolic diseases such as obesity and type 2 diabetes (DM2) [39]. Short-term antibiotics use can lead to long-term disorder of intestinal flora, which will result in the occurrence or aggravation of the diseases [40].

The researchers have investigated the associations between antibiotic use and childhood asthma, allergies, and body mass index changes [41]. Small intestine bacterial and coliform have a great influence on weight-regulating system and glucose homeostasis [42]. Another team discovered that macrolide use was positively relevant to asthma and overweight [15]. The possibility of suffering from asthma and overweight on children who had frequent macrolide use during the first 2 years of life increased significantly. In addition, the overweight children with asthma had unique microbiota. Odds ratio for the group that received 42 macrolide courses compared with the non-exposed was 6.11. Other results showed that macrolide use in childhood is associated with long-term distortions in the composition, function and antibiotic resistance of the intestinal microbiota.

Early exposure to antibiotics has been an important reason which causes childhood obesity [43]. Paolella and Vajro proposed three methods to cope with the impact caused by antibiotics: First, a more judicious use of antibiotics and for instance, the use of narrowerspectrum molecules for simple bacterial infections would surely be advantageous. Second, increase intake of $\omega-3$ fatty acids. Finally, administration of prebiotics or probiotics is another possible strategy in this setting [44].

\section{Gut microbiota carried antibiotic resistance gene}

An increased incidence of antibiotic resistance genes observed in antibiotic-exposed children after treatment [29]. The presence of antibiotic resistance genes rose sharply and rapidly during antibiotic treatment and then declined swiftly after antibiotic therapy, whereas the antibiotic resistance genes still presented after antibiotic therapy discontinued for much longer periods of time. More interestingly, the researchers also observed that some children harboured antibiotic resistance genes as early as 2 months of age, before any antibiotic treatments. In recent report, researchers from Washington University School of Medicine (WUSM) demonstrated that gut microbiota in healthy American children also carried a large number of antibiotic resistance genes, likely to make harmful effects [32].

Nonetheless, to date, it has remained unclear where antibiotic resistance genes originated. Some previous studies have determined that some new-born infants harboured antibiotic resistance genes, potentially acquired from their mothers before their birth $[45,46]$. However, the reason for the increased abundance of antibiotic resistance genes is still 
unknown. Here are the possible explanations: (i) response to antibiotics passed on to the child from the environment (for example, mothers' breast milk), (ii) a response to a natural antibiotic molecule produced by other gut bacteria, (iii) pre-existing presence of the antibiotic resistance gene in the genomes of some early inhabitants of the gut [47], (iv) the spread of antibiotic resistance through natural populations due to widespread use of antibiotics [29]. Additional studies will be needed to find out the exact reasons for the early presence of antibiotic resistance genes in the gut microbiota of infants. Furthermore, it is particularly significant to understand the effects of childhood antibiotic exposure on the presence of antibiotic resistance genes.

\section{Mechanism of intestinal drug resistant bacteria on infants}

The incidence of antibiotics-resistant genes rises rapidly during antibiotic treatment then drop quickly after treatment was discontinued. But resistance genes encoded on small DNA molecules called mobile elements, one means by which resistance genes can be transmitted among bacteria, persisted much longer after antibiotic withdrawal [29]. After macrolide antibiotics treatment, in the infants' body, macrolide antibiotics drug resistance increased rapidly, namely, macrolide antibiotics promoted the production of antibiotic resistance. Based on the metagenomic analysis, one recent study discovered that macrolide resistance was high in the recently macrolide-exposed gut microbial, and bile-salt hydrolases were reduced [15]. Bile-salt metabolism (BSM) is an important function of intestinal microbes, which influences energy and metabolism of the host [48]. Frequent antibiotic intake will accelerate the production of antibiotic resistance in infants, particularly those born prematurely, because they received early and often extensive antibiotic therapy in the first months after birth [32]

\section{Brief summary and Future prospects}

Gut microbiota plays an important role in nutrient absorption, immune system development and metabolic homeostasis of the human body $[49,50]$. Antibiotics are among the many factors that influence the development of the microbiota and health [51].

In recent years, the research on gut microbiota of infants, particularly antibiotic-treated infants, has received more attention. Antibiotics use is detrimental to host health in the short and the long-term. In this review, to better understand the impacts of early-life antibiotic use on gut microbiota and health of infants, we showed: (i) antibiotics use leads to remarkable changes in the structure of gut microbiota and metabolic capacity, (ii) antibiotic exposures impair the diversity and stability of gut microbiota, (iii) antibiotic treatment increases the risk of diseases, such as inflammatory bowel disease (IBD), obesity, diabetes and asthma, as well as allergies, (iv) antibiotic resistance genes rise sharply and rapidly during antibiotic treatment and then declined swiftly after antibiotic therapy, whereas the antibiotic resistance genes still presented after antibiotic therapy discontinued for much longer periods of time. Meanwhile, we also investigate mechanism of intestinal drug resistant bacteria from some recent researches.

Because antibiotics are frequently used during the first critical years of gut microbiota development. Antibiotic use during childhood is prevalent in most parts of the world [52]. The average U.S. child receives about three courses of antibiotic by the age of 2 and 10 courses by the age of 10 [53]. Furthermore, the effectiveness of antibiotics has been weaken since they were introduced into modern medicine more than 70 years ago [54]. One important consequence of antibiotic usage is the spread of antibiotic resistance genes, which is a serious public health issue. Antibiotic resistance is of major public health concern globally and is expected to become an increasingly serious obstacle in the treatment. In an earlier review, it was shown that, on present trends, the annual global death toll from bacterial resistance would be more than ten million, a figure more significant than that die from cancer [55]. Furthermore, bacteria, viruses and other microbes will again evolve resistance [56]. It has been demonstrated that macrolide use promoted a marked increase in macrolide resistance of the microbiota [15]. And worse still, abuse and misuse of antibiotics make antibiotic resistance give birth to the 'super bacteria' [57,58]. In one study on Mycobacterium tuberculosis, the causative agent of tuberculosis, Warrier et al. [59] have discovered a novel bacterial drug resistance, antibiotic inactivation via $\mathrm{N}$-methylation. Nevertheless, it is not clear whether this new drug resistance mechanism is widely present in bacteria, and how to inhibit bacterial drug resistance $[58,59]$. Currently, the UN General Assembly High-Level Meeting of Heads of State discussed sustainable access to effective antimicrobials in September, 2016. International scientists advocated awareness about lack of access to antibiotics and drug resistance [60].

Despite widespread use of antibiotics in children, the effects of antibiotic. With our growing appreciation of gut microbiota's role in human health and how to maintain healthy gut microbiota, it is particularly critical to understand the short-and long-term effects of antibiotic treatments on the gut microbiota. As we look to the future, additional studies will be needed to find out the effects of childhood antibiotic exposure on gut microbiota and health. Last but not least, little is currently known about mechanism of intestinal drug resistant bacteria in infants, but, it's certainly the case that antibiotic use will have a significant impact on gut microbiota of infants. Therefore, to better understand the health implications of early-life antibiotic use, mechanism of infant's intestinal drug resistant bacteria is another issue that requires further investigation and prompt attention.

\section{References}

1. Neu J (2015) Developmental aspects of maternal-fetal and infant gut microbiota and implications for long-term health. Matern Health Neonatol Perinatol 1: 1-7.

2. Hadhazy $A$ (2010) Think twice how the gut's second brain influences mood and well-being. Sci Am 2: 1-3.

3. Li J, Jia H, Cai X, Zhong H, Feng Q, et al. (2014) An integrated catalog of reference genes in the human gut microbiome. Nat Biotechnol 32: 834-841.

4. Sekirov I, Russell SL, Antunes LC, Finlay BB (2010) Gut microbiota in health and disease. Physiol Rev 90: 859-904.

5. Clemente JC, Ursell LK, Parfrey LW, Knight R (2012) The impact of the gut microbiota on human health: An integrative view. Cell 148: 1258-1270.

6. Virta L, Auvinen A, Helenius H, Huovinen P, Kolho KL (2012) Association of repeated exposure to antibiotics with the development of pediatric Crohn's disease--a nationwide, register-based Finnish case-control study. Am J Epidemiol 175: 775-784.

7. Chai G, Governale L, McMahon AW, Trinidad JP, Staffa J, et al. (2012) Trends of outpatient prescription drug utilization in US children, 2002-2010. Pediatrics 130: 23-31.

8. Aloisio I, Quagliariello A, Fanti SD, Luiselli D, Filippo CD, et al. (2016) Evaluation of the effects of intrapartum antibiotic prophylaxis on new-born intestinal microbiota using a sequencing approach targeted to multi hypervariable $16 \mathrm{~S}$ rDNA regions. Appl Microbiol Biotechnol 100: 5537-5546.

9. Bäumler AJ, Sperandio $\vee(2016)$ Interactions between the microbiota and pathogenic bacteria in the gut. Nature 535: 85-93.

10. Thaiss CA, Zmora N, Levy M (2016) The microbiome and innate immunity. Nature 535: 65-74

11. Vikram A,Kim YR (2016) Vascular microRNA-204 is remotely governed by the microbiome and impairs endothelium-dependent vasorelaxation by downregulating Sirtuin1. Nat Commun 7: 12565. 
Citation: Hong YW, Si TS, Zhen YY, Xiu YW, Guo ZW (2017) Impact of Early-Life Antibiotic Use on Gut Microbiota of Infants. J Microb Biochem Technol 9:227-231. doi: 10.4172/1948-5948.1000369

12. Paul J, Kennedy AM, John FC, Paul RR, Timothy G (2016) Dinan, catherine stanton, microbiome in brain function and mental health. Trends Food Sci Tech 5: 1-13.

13. Matamoros S, Gras-Leguen C, Le Vacon F, Potel G, de La Cochetiere MF (2013) Development of intestinal microbiota in infants and its impact on health. Trends Microbiol 21: 167-173.

14. Human Microbiome Project Consortium (2012) Structure, function and diversity of the healthy human microbiome. Nature 486: 207-214.

15. Korpela K, Salonen A, Virta LJ, Kekkonen RA, Forslund K, et al. (2016) Intestinal microbiome is related to lifetime antibiotic use in Finnish pre-school children. Nat Commun 7: 1258-1270.

16. Pamer EG (2016) Resurrecting the intestinal microbiota to combat antibioticresistant pathogens. Science 352: 535-538.

17. Ponziani FR, Scaldaferri F, Petito V, Paroni Sterbini F, Pecere S, et al. (2016) The role of antibiotics in gut microbiota modulation: The eubiotic effects of rifaximin. Dig Dis 34: 269-278

18. Klemm E, Dougan G (2016) Advances in understanding bacterial pathogenesis gained from whole-genome sequencing and phylogenetics. Cell Host Microbe 19: $599-610$.

19. Bokulich NA, Chung J, Battaglia T, Henderson N, Jay M, et al. (2016) Antibiotics, birth mode and diet shape microbiome maturation during early life. Sci Trans Med 8: 1-13

20. Nobel YR, Cox LM, Kirigin FF, Bokulich NA, Yamanishi S, et al. (2015) Metabolic and metagenomic outcomes from early-life pulsed antibiotic treatment. Nat Commun 6: 7486 .

21. Subramanian S, Huq S, Yatsunenko T, Haque R, Mahfuz M, et al. (2014) Persistent gut microbiota immaturity in malnourished Bangladeshi children. Nature 510: 417-421.

22. Dethlefsen L, Relman DA (2011) Incomplete recovery and individualized responses of the human distal gut microbiota to repeated antibiotic perturbation. Proc Natl Acad Sci U S A 108: 4554-4561.

23. Jernberg C, Lofmark S, Edlund C, Jansson JK (2007) Long-term ecologica impacts of antibiotic administration on the human intestinal microbiota. ISME J 1: 56-66.

24. Rutten NBMM, Rijkers GT, Meijssen CB, Crijns CE, Oudshoorn JH, et al. (2015) Intestinal microbiota composition after antibiotic treatment in early life: The INCA study. BMC Pediatr 15: 204

25. Cox LM, Yamanishi S, Sohn J, Alekseyenko AV, Leung JM, et al. (2014) Altering the intestinal microbiota during a critical developmental window has lasting metabolic consequences. Cell 158: 705-721.

26. Sonnenburg JL, Bäckhed F (2016) Diet-microbiota interactions as moderators of human metabolism. Nature 535: 56-64.

27. Cani PD (2016) Gut microbiota: Changes in gut microbes and host metabolism Squaring the circle? Nat Rev Gastroenterol Hepatol 13: 563-564.

28. Pitt JM, Waldschmitt N, Kroemer G, Chamaillard M, Boneca IG, et al. (2016) Fine-tuning cancer immunotherapy: Optimizing the gut microbiome. Cancer res 76: 1-6.

29. Yassour M, Vatanen T, Siljander H, Hämäläinen AM, Härkönen T, et al. (2016) Natural history of the infant gut microbiome and impact of antibiotic treatment on bacterial strain diversity and stability. Sci Transl Med 8: 343ra81.

30. Bisgaard H, Li N, Bonnelykke K, Chawes BL, Skov T, et al. (2011) Reduced diversity of the intestinal microbiota during infancy is associated with increased risk of allergic disease at school age. J Allergy Clin Immunol 128: 1-5.

31. Kronman MP, Zaoutis TE, Haynes K, Feng R, Coffin SE (2012) Antibiotic exposure and IBD development among children: A population-based cohort study. Pediatrics 130: 794-803.

32. Gasparrini AJ, Crofts TS, Gibson MK, Tarr PI (2016) Antibiotic perturbation of the preterm infant gut microbiome and resistome. Gut Microbes 7: 443-449.

33. Gibson MK, Wang B, Ahmadi S (2016) Developmental dynamics of the preterm infant gut microbiota and antibiotic resistome. Nat Microbiol 1: 16024.

34. Bailey LC, Forrest CB, Zhang P, Richards TM, Livshits A, et al. (2014)
Association of antibiotics in infancy with early childhood obesity. JAMA Pediatr 168: 1063-1069.

35. Saari A, Virta LJ, Sankilampi U, Dunkel L, Saxen H (2015) Antibiotic exposure in infancy and risk of being overweight in the first 24 months of life. Pediatrics 135: $617-626$.

36. Trasande L, Blustein J, Liu M, Corwin E, Cox LM, et al. (2013) Infant antibiotic exposures and early-life body mass. Int J Obes (Lond) 37: 16-23.

37. Penders J, Kummeling I, Thijs C (2011) Infant antibiotic use and wheeze and asthma risk: A systematic review and meta-analysis. Eur Respir J 38: 295-302.

38. Albenberg L, Kelsen $\mathrm{J}$ (2016) Advances in gut microbiome research and relevance to pediatric diseases. J Pediatr 178: 16-23.

39. Hartstra AV, Nieuwdorp M, Herrema H (2016) Interplay between gut microbiota, its metabolites and human metabolism: Dissecting cause from consequence. Trends in Food Sci Tech 8: 1-11.

40. Lange K, Buerger M, Stallmach A, Bruns T (2016) Effects of antibiotics on gut microbiota. Dig Dis 34: 260-268.

41. Foliaki S, Pearce N, Bjorksten B, Mallol J, Montefort S, et al. (2009) Antibiotic use in infancy and symptoms of asthma, rhinoconjunctivitis and eczema in children 6 and 7 years old: International study of asthma and allergies in childhood phase III. J Allergy Clin Immunol 124: 982-989.

42. Scheithauer TPM, Dallinga-Thie GM, Vos WMD, Nieuwdorp M, Raalte DHV (2016) Causality of small and large intestinal microbiota in weight regulation and insulin resistance. Mol Metab 5: 759-770.

43. Korpela K, Salonen A, Virta LJ, Kekkonen RA, de Vos WM (2016) Association of early-life antibiotic use and protective effects of breast-feeding: Role of the intestinal microbiota. JAMA Pediatr 170: 750-757.

44. Paolella G, Vajro P (2016) Childhood obesity, breastfeeding, intestina microbiota and early exposure to antibiotics: What is the link? JAMA Pediatr 170: $735-737$

45. Alicea-Serrano AM, Contreras M, Magris M, Hidalgo G, Dominguez-Bello MG (2013) Tetracycline resistance genes acquired at birth. Arch Microbiol 195: 447 451

46. Fouhy F, Ogilvie LA, Jones BV, Ross RP, Ryan AC, et al. (2014) Identification of aminoglycoside and $B$-lactam resistance genes from within an infant gut functional metagenomic library. PLoS ONE 9: e108016.

47. Clemente JC, Pehrsson EC, Blaser MJ, Sandhu K, Gao Z, et al. (2015) The microbiome of uncontacted Amerindians. Sci Adv 1: e1500183.

48. Joyce SA, Macsharry J, Casey PG, Kinsella M, Murphy EF, et al. (2014) Regulation of host weight gain and lipid metabolism by bacterial bile acid modification in the gut. Proc Natl Acad Sci U S A.111: 7421-7426.

49. Roeselers G, Bouwman J, Levin E (2016) The human gut microbiome, diet and health: "Post hoc non ergo propter hoc". Trends in Food Sci Tech 8: 1-4

50. Zeng MY, Inohara N, Nuñez G (2017) Mechanisms of inflammation-driven bacterial dysbiosis in the gut. Mucosal Immunol 10: 18-26.

51. Blaser MJ (2016) Antibiotic use and its consequences for the normal microbiome. Science 352: 544-545

52. Fouhy F, Guinane CM, Hussey S, Wall R, Ryan CA, et al. (2012) High throughput sequencing reveals the incomplete, short-term recovery of infant gut microbiota following parenteral antibiotic treatment with ampicillin and gentamicin. Antimicrob Agents Chemother 56: 5811-5820.

53. Hicks LA, Taylor TH Jr, Hunkler RJ (2013) More on U.S. outpatient antibiotic prescribing, 2010. N Engl J Med 369: 1175-1176.

54. Jørgensen PS, Wernli D, Carroll SP, Dunn RR, Harbarth S, et al. (2016) Use antimicrobials wisely. Nature 537: 159-161.

55. Matthiessen L, Bergström R, Dustdar S, Meulien P, Draghia-Akli R (2016) Increased momentum in antimicrobial resistance research. Lancet 388: 865

56. Carroll SP, Jorgensen PS, Kinnison MT, Bergstrom CT, Denison RF, et al. (2014) Applying evolutionary biology to address global challenges. Science 346: 1245993.

57. Laxminarayan R, Sridhar D, Blaser M, Wang M, Woolhouse M (2016) Achieving global targets for antimicrobial resistance. Science 353: 874-875. 
Citation: Hong YW, Si TS, Zhen YY, Xiu YW, Guo ZW (2017) Impact of Early-Life Antibiotic Use on Gut Microbiota of Infants. J Microb Biochem Technol 9:227-231. doi: 10.4172/1948-5948.1000369

58. Malone KM, Gordon SV (2016) Antibiotic methylation: A new mechanism of antimicrobial resistance. Trends Microbiol 24: 771-772.

59. Warrier T, Kapilashrami K, Argyrou A, loerger TR, Little D, et al. (2016) $\mathrm{N}$-methylation of a bactericidal compound as a resistance mechanism in Mycobacterium tuberculosis. Proc Natl Acad Sci U S A 113: E4523-30.
60. Laxminarayan R, Amábile-Cuevas CF, Cars O, Evans T, Heymann DL, et al (2016) UN high-level meeting on antimicrobials - What do we need? Lancet 388: 218-220. 